Effects of the scalar dissipation rate on the steady laminar flamelet model

This article has been downloaded from IOPscience. Please scroll down to see the full text article.

2010 Phys. Scr. 2010014048

(http://iopscience.iop.org/1402-4896/2010/T142/014048)

View the table of contents for this issue, or go to the journal homepage for more

Download details:

IP Address: 159.226.230.152

The article was downloaded on 05/01/2011 at 01:56

Please note that terms and conditions apply. 


\title{
Effects of the scalar dissipation rate on the steady laminar flamelet model
}

\author{
Jian Zhang, Guodong Jin and Guowei He \\ LNM, Institute of Mechanics, Chinese Academy of Sciences, Beijing, People's Republic of China \\ E-mail: hgw@lnm.imech.ac.cn
}

Received 26 October 2009

Accepted for publication 2 June 2010

Published 31 December 2010

Online at stacks.iop.org/PhysScr/T142/014048

\begin{abstract}
The effects of the scalar dissipation rate (SDR) on the quenching points of the steady laminar flamelet model at different stoichiometric ratios of reactants are investigated for a one-step reversible reaction with the Arrhenius rate. SDRs are either presumed uniform or modeled by the mapping closure approach. With these two models, steady flamelet equations are solved in mixture-fraction space, and complete 'S-shaped' curves are obtained. The results show that the SDR models affect the locus of the 'S-shaped' curves and the quenching points are remarkably different. The quenching state is reached at a lower $\chi_{\mathrm{st}}$ for $\chi=\chi_{\mathrm{st}}$ but at a higher $\chi_{\mathrm{st}}$ for $\chi=\chi_{\mathrm{st}} \exp \left[-2 \mathrm{erfc}^{-1}(2 z)^{2}\right]$. However, when the flame state is far from the quenching point, the difference in 'S-shaped' curves is small. This result is associated with the mixed state of the reactants; the poorer the mixing, the more remarkable the difference in 'S-shaped' curves becomes.
\end{abstract}

PACS numbers: 47.27.E-, 47.70.pq

(Some figures in this article are in colour only in the electronic version.)

\section{Introduction}

The steady laminar flamelet model (SLFM) is one of the most popular models used in non-premixed combustion. The idea that a turbulent diffusion flame consists of an ensemble of stretched laminar flamelets came from Williams [1]. Flamelets are thin reactive-diffusive layers embedded in an otherwise non-reacting turbulent flow field. In practice, flamelets occur in many different combustion apparatuses, such as the diesel engine, the spark-ignition engine, supersonic combustion, etc [1]. A flamelet equation has the mixture fraction as an independent variable and uses the scalar dissipation rate (SDR) for the mixing process. Therefore, the SDR plays a central role in the flamelet model in non-premixed combustion. In general, flamelets are relatively insensitive to variations in scalar dissipation and thus scalar dissipation can be assumed to be a constant mean value [2]. However, it was found that the SDR has a significant effect on extinction and ignition in non-premixed combustion [3]. Therefore, it is necessary to investigate the effects of SDR models on the solutions of flamelet equations. It is expected that this will help in understanding the turbulence-chemistry interaction and in extending the SLFM.

\section{A simple model for chemical reactions}

We consider a one-step reversible reaction as given below:

$$
F+r O \rightleftharpoons(r+1) P,
$$

where $F, O$ and $P$ are fuel, oxidant and product, respectively.

For a simple reaction of two reactants, we define the mixture fraction by

$$
z=\frac{r Y_{F}-Y_{O}+1}{1+r} .
$$

Here $r$ is the stoichiometric ratio and is defined as the mass of the oxidant disappearing with unit mass of fuel [4]. Therefore, the stoichiometric value of the mixture fraction is

$$
z_{\mathrm{st}}=\frac{1}{1+r}
$$

The subscript st indicates the stoichiometric value. $Y_{F}, Y_{O}$ and $Y_{P}$ are the mass fractions of the fuel, oxidant and product, respectively. We set $r=1$ and $r=2$, which correspond to the values of $z_{\text {st }}$ in table 1 . Using an equilibrium constant $K$, the 
Table 1. Stoichiometric ratios used in this paper.

\begin{tabular}{lcc}
\hline Stoichiometric ratio $(r)$ & 1 & 2 \\
Stoichiometric mixture fraction $\left(z_{\mathrm{st}}\right)$ & 0.5 & 0.333 \\
\hline
\end{tabular}

Table 2. Numerical values of the thermochemistry parameters.

\begin{tabular}{cccc}
\hline $\mathrm{A}$ & $\alpha$ & $\beta$ & $\mathrm{K}$ \\
\hline $8 \times 10^{4}$ & 0.87 & 4.0 & 100 \\
\hline
\end{tabular}

production rate in the simple reaction can be written as

$$
\begin{aligned}
\omega_{\mathrm{p}}= & (r+1) A \exp \left(\frac{-\beta}{\alpha}\right) \\
& \times \exp \left[\frac{-\beta(1-\theta)}{1-\alpha(1-\theta)}\right]\left(Y_{F} Y_{O}-\frac{1}{K} \theta^{r+1}\right) .
\end{aligned}
$$

Here, the chemical reaction is characterized by the pre-exponential factor $A$, the heat-release parameter $\alpha$ and the Zeldovich number $\beta$. The latter two parameters are defined as

$$
\begin{gathered}
\alpha=\frac{T_{\mathrm{b}}-T_{\mathrm{u}}}{T_{\mathrm{b}}}, \\
\beta=\frac{T_{\mathrm{a}}}{T_{\mathrm{b}}} \alpha,
\end{gathered}
$$

where $T_{\mathrm{a}}$ is the activation temperature, $T_{\mathrm{b}}$ is the adiabatic flame temperature and $T_{\mathrm{u}}$ is the temperature of both the unburned fuel and the oxidant. The normalized temperature $\theta$ is defined as

$$
\theta=\frac{T-T_{\mathrm{u}}}{T_{\mathrm{b}}-T_{\mathrm{u}}}
$$

Table 2 shows the value of thermochemistry parameters used in this study. These values are selected so that the reaction rates are strongly temperature dependent and lead to local extinction in a strongly burning flame $[4,5]$. For a one-step reaction, each mass fraction together with the mixture fraction determines the other two mass fractions. The product mass fraction, $Y_{\mathrm{P}}$, is equal to the normalized temperature $\theta$ [4].

\section{The steady laminar flamelet equations}

The steady laminar flamelet equations can be described as

$$
0=\frac{\chi}{2} \frac{\mathrm{d}^{2} Y_{i}}{\mathrm{~d} z^{2}}+\omega, \quad i=F, O, P .
$$

Here, $Y_{i}$ are mass fractions of the reactants. $\chi$ is the dissipation rate of the mixture fraction, that is, $\chi=2 D(\nabla z)^{2}$. In the SLFM, mass fractions depend on space and time through the local instantaneous value of the mixture fraction $z$ and $\operatorname{SDR} \chi$. The $\chi$ acts as an external parameter that is imposed on the flamelet structure by the mixture fraction [6]. This suggests that there is a general dependence of $\chi$ on the mixture fraction. This dependence is the so-called 'scalar dissipation rate (SDR) model'. We investigate two different SDR models: the first one is the mapping closure model [7, 8] and the second one is uniform in both space and time. The two models are given in table 3 .
Table 3. The SDR model.

\begin{tabular}{cc}
\hline & The SDR model \\
\hline Model 1 & $\chi=\chi_{\mathrm{st}} \exp \left[-2 \operatorname{erfc}^{-1}(2 z)^{2}\right]$ \\
Model 2 & $\chi=\chi_{\mathrm{st}}$ \\
\hline
\end{tabular}

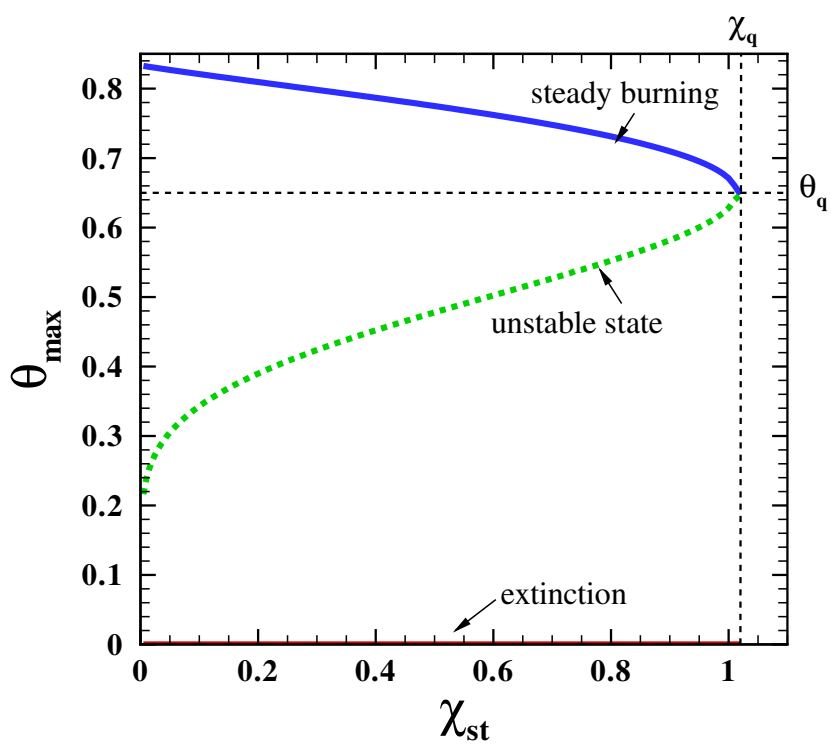

Figure 1. The typical 'S-shaped' curve with Arrhenius kinetics.

These two models will be used to investigate the effects of SDR models on the solutions of the SLFM. The complete locus of solutions of steady flamelet equations should be calculated. With Arrhenius kinetics, there are typically three flame states: steady burning, the unstable, partially extinguished state and complete extinction. The curve, sometimes called the 'S-shaped' curve in diffusion flame theory, is determined primarily by the chemical kinetics [9]. A typical curve is shown in figure 1 . The vertical axis is maximum flame temperature $\theta_{\max }$ and the horizontal axis is SDR at the stoichiometric value of the mixture fraction, $\chi_{\text {st }}$. Figure 1 indicates that on the steady burning branch, the maximum flame temperature decreases with increasing dissipation rate. When the critical point is reached, the flame temperature becomes so low that Arrhenius rate factors in the chemical kinetics begin to limit the reaction rates. Below the critical point on the unstable branch, the dissipation rate must decrease with decreasing flame temperature, and the flame state is unstable, without a steady flame; on the complete extinction line, the effect of chemical kinetics is negligible. Therefore, the location of the critical point is very important for the unstable flame state. At the critical point, the maximum temperature is $\theta_{\mathrm{q}}$ and the SDR is $\chi_{\mathrm{q}}$, where the subscript $\mathrm{q}$ indicates the value at the quenching point in the SLFM, i.e. the critical point in this paper.

\section{Results and discussion}

Equation (8) can be discretized as a large, nonlinear system of equations. But it is noteworthy that this system has multiple roots. There are some difficulties in solving equation (8), but in order to obtain the complete S-shaped curve, all solutions of the system should be obtained. 
Table 4. Calculation results.

\begin{tabular}{cllcc}
\hline \multirow{2}{*}{$\begin{array}{c}\text { Stoichiometric } \\
\text { ratio }\end{array}$} & & \multicolumn{3}{c}{$\chi_{\mathrm{q}}$ and $\theta_{\mathrm{q}}$} \\
\cline { 3 - 5 } & SDR model & This work & {$[10]$} & [4] \\
\hline$r=1$ & $\begin{array}{l}\text { Model 1 } \\
\text { (reference) }\end{array}$ & $1.02,0.65$ & $1.02,0.65$ & $1.04,0.65$ \\
& Model 2 & $0.96,0.65$ & - & - \\
$r=2$ & Model 1 & $1.54,0.66$ & - & - \\
& Model 2 & $1.35,0.66$ & - & - \\
\hline
\end{tabular}

In this work we used the BVP4C MATLAB program to solve the flamelet equations. The results obtained are listed in table 4 . We use the first case as a reference case. The chemical reaction, thermochemistry parameters, stoichiometric ratio and SDR model are the same as those in $[4,10]$. Here the values of $\chi_{\mathrm{q}}$ and $\theta_{\mathrm{q}}$ are consistent with [10] and very close to those in [4].

In table 4, one can see different chemical states at the quenching point that result from the SDR models. The different SDR models may cause the differences in stoichiometric dissipation rate at the quenching point $\chi_{\mathrm{q}}$, especially in the $r=2$ case where the difference is more remarkable. However, the maximum temperatures at the quenching point $\theta_{\mathrm{q}}$, which were related to thermochemistry parameters such as $\alpha$ and $\beta$ [4], in table 1, are insensitive to the SDR model.

As can be seen in figures 2(a) and 3(a), the complete locus of solutions of steady flamelet equations is plotted for different stoichiometric ratios using the SDR models. From them, we can see the effects of the SDR models on S-shaped curves. Figures 2(b) and 3(b) show the distributions of temperature in the mixture fraction space, i.e. the solutions of the steady flamelet equations. The solid lines correspond to the steady burning state, and the dashed lines to the unstable state. We have chosen dissipation rates $\chi_{\mathrm{st}}=0.9$ in figure 2(b) and $\chi_{\mathrm{st}}=1.2$ in figure $3(\mathrm{~b})$ for presenting the solutions of the flamelet equations. Figure 2 shows that, for $r=1$, the SDR model has an effect on the quenching point of the S-shaped curve. Near the quenching point, the solutions of the flamelet equations for the two SDR models are remarkably different (see figure 2(b)), which was more obvious for $r=2$ (see figure $3(\mathrm{~b})$ ). The quenching point is a critical point and is very important for the SLFM. The steady-flamelet library is shown in figure 4, which indicates that, in the SLFM, the steady-flamelet library is somehow incomplete because it could represent any of the 'partially extinguished' intermediate states that should fill the gap between the critical point and complete extinction [9]. Hence, the critical point determines whether the flame state is steady burning or complete extinction. The different quenching points indicate the effects of the SDR model on the simulation of the flame state.

The quenching point was determined based on two quantities: the maximum temperature and the dissipation rate of the mixture fraction at a stoichiometric value. In model 1 , the dissipation rate is not uniform and the maximum dissipation rate is $\chi_{\mathrm{st}}$. However, in model 2 , the dissipation rate is uniformly $\chi_{\mathrm{st}}$. On average, the dissipation effect of model 2 was higher than that of model 1 . As we know, the maximum temperature at the quenching point is related to
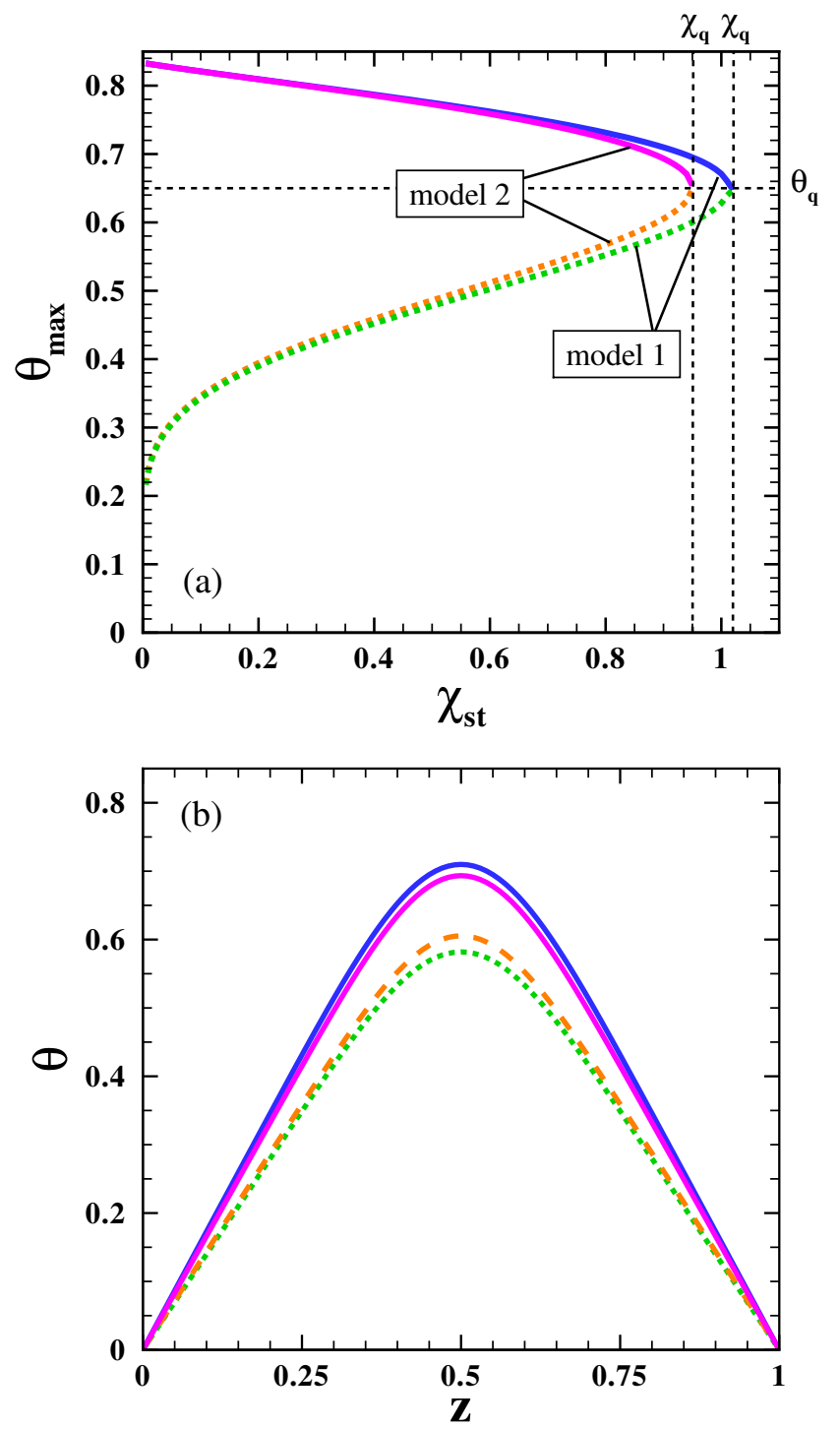

Figure 2. Stoichiometric ratio $r=1$ : (a) Locus of maximum flame temperature from a complete set of steady-flamelet solutions for two SDR models. Model 1 is a mapping closure model and model 2 is a uniform model. (b) The distributions of temperature in the mixture fraction space. The solid lines correspond to the steady burning states (the upper line-model 1, the lower line-model 2), and the dashed lines to the unstable states (the upper line-model 2 , the lower line-model 1).

thermochemistry parameters such as $\alpha$ and $\beta$ but insensitive to the SDR model. Hence, we have fixed the value of the maximum temperature at the quenching point in this paper. The higher dissipation effect implies better mixing; the flame temperature at the steady burning state is more inclined towards the quenching temperature with increasing maximum dissipation rate. In particular, when the flame is near the quenching state, the mixture fraction field mixes poorly and the dissipative effect of the non-uniform SDR model is considerably lower than that of the uniform SDR model; hence, the quenching point is clearly different, and the quenching point of the S-shaped curve with model 2 is on the left hand side. When the flame is far away from the quenching state, the mixture fraction field is well mixed; even if the non-uniform SDR model is applied, all values of the dissipation rate are close to the maximum dissipation rate, and 

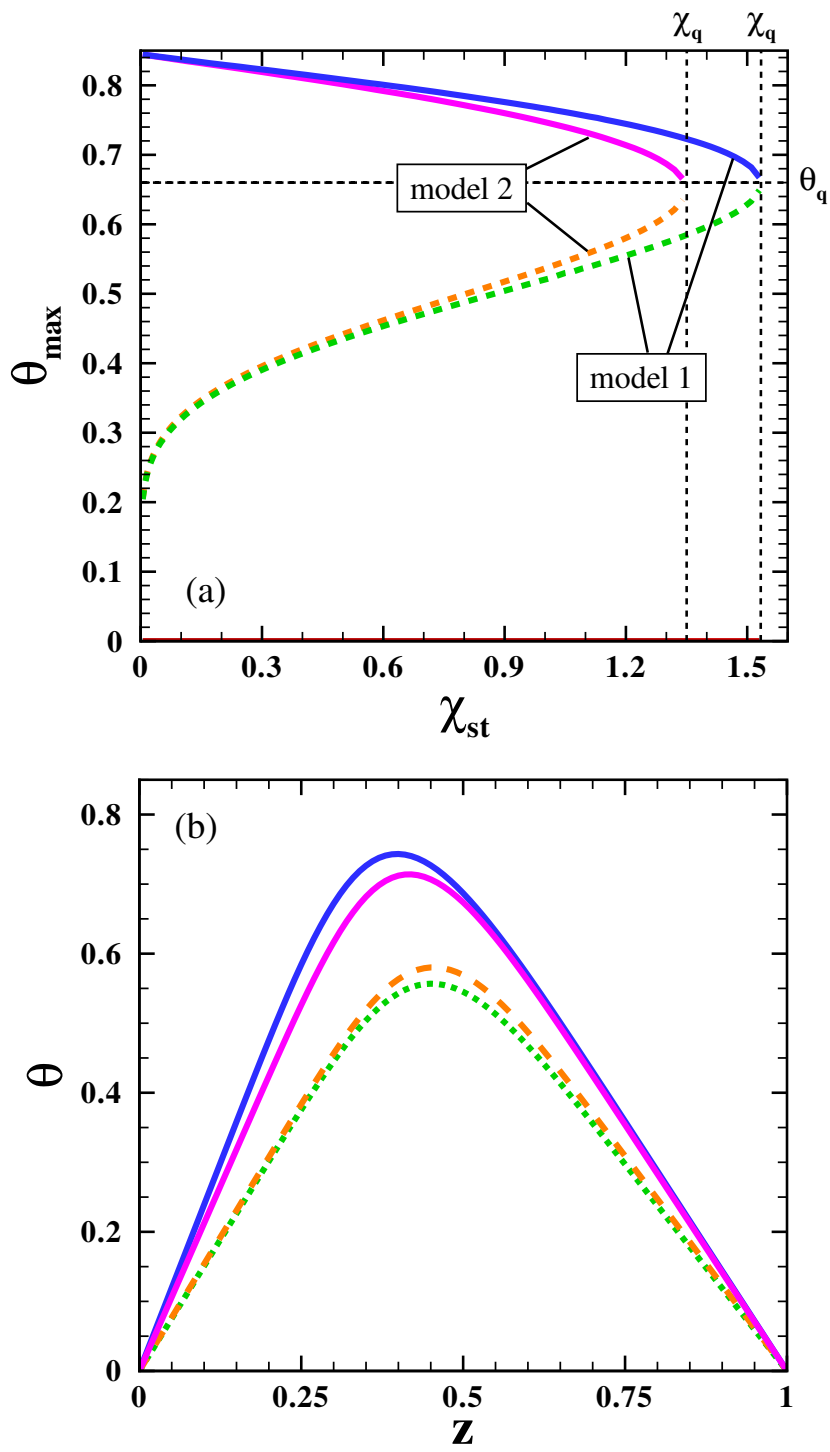

Figure 3. Stoichiometric ratio $r=2$ : (a) Locus of the maximum flame temperature from a complete set of steady-flamelet solutions for two SDR models. Model 1 a mapping closure model and model 2 is a uniform model. (b) The distributions of temperature in the mixture fraction space. The solid lines correspond to the steady burning states (the upper line-model 1, the lower line-model 2), and the dashed lines to the unstable states (the upper line-model 2, the lower line-model 1).

so the dissipation effects of the non-uniform SDR model and the uniform SDR model are similar to each other. Therefore, the S-shaped curves for the two models are remarkably different near the quenching state and similar far away from the quenching state.

\section{Conclusion}

In the SLFM, the SDR can be regarded as a parameter to indicate the effect of flow on mixing and describe the influence of the flow field on the flamelet structure. SDR plays a central role and affects the performance of the SLFM. We used the BVP4C MATLAB program to solve the flamelet equations with Arrhenius-type chemical reaction and obtained complete S-shaped curves. In the reference

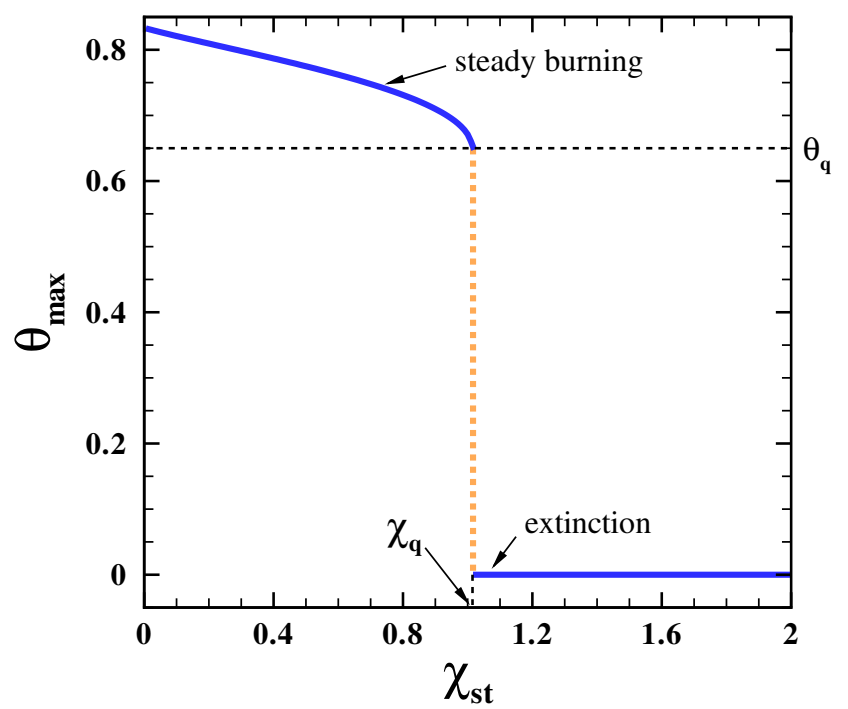

Figure 4. Locus of maximum flame temperatures in the steady-flamelet library. There is a discontinuous jump between burning and extinguished solutions at the critical point. This should be compared with figures 2 and 3 .

case (see table 4), our results were in good agreement with $[4,10]$.

This paper shows that the SDR model influences the locus of the S-shaped curve. Since the quenching temperature was a fixed value for different SDR models and the higher dissipative effect of SDR models would accelerate the drop in temperature from a high temperature at the steady burning state, the quenching state was reached at a lower $\chi_{\text {st }}$ for the uniform SDR model but at a higher $\chi_{\text {st }}$ for the non-uniform SDR model. The difference in S-shaped curves was considerable near the quenching point and very slight where the state was far away from the quenching point, which was associated with the mixed state of the reactants. As the mixing became poorer, the difference in S-shaped curves became more remarkable.

As we know, the quenching point is a critical point and determines whether the flame state is burning or extinction. From the above analysis, if the SLFM was used to calculate the flame extinction, the SDR model would play an important role and remarkably affect the calculation results. An accurate SDR model is necessary for flame extinction.

\section{Acknowledgments}

This work was supported by CAS (KJCX2-SW-L08), 973 Program of China (2007CB814800 and 2009CB724100), NSFC (10702074, 10732090 and 50906096) and the LNM initial funding for young investigators.

\section{References}

[1] Williams F A 2000 Prog. Energy Combust. Sci. 26657

[2] Bilger R W, Pope S B, Bray K N C and Driscoll J F 2005 Proc. Combust. Inst. 3021

[3] Pitsch H and Fedotov S 2001 Combust. Theor. Model. 541

[4] Sripakagorn P, Mitarai S, Kosály G and Pitsch H 2004 J. Fluid Mech. 518231 
[5] de Bruyn Kops S M 1999 Numerical simulations of non-premixed turbulent combustion PhD Thesis University of Washington, Seattle

[6] Peters N 2000 Turbulent Combustion (Cambridge: Cambrige University Press)

[7] O'Brien E E and Jiang T L 1991 Phys. Fluids A 33121
[8] He G W and Zhang Z F 2004 Phys. Rev. E 70036309

[9] Pierce C D and Moin P 2004 J. Fluid Mech. 50473

[10] Sripakagorn P, Kosály G and Pitsch H 2000 Annual Research Briefs 2000 Center for Turbulence Research, Stanford University/NASA Ames pp 117-28 